\title{
A Common Fixed Point Theorem for $R$-Weakly Commuting Maps Satisfying Property (E.A.) in Fuzzy Metric Spaces Using Implicit Relation
}

\author{
Saurabh Manro \\ School of Mathematics and Computer Applications, Thapar University \\ Patiala (Punjab) \\ Email: sauravmanro@hotmail.com, sauravmanro@yahoo.com
}

\begin{abstract}
The purpose of this paper is to prove a common fixed theorem for $R$-weakly commuting mappings via an implicit relation in fuzzy metric space. While proving our result, we utilize the idea of property (E.A.) due to Aamri and El. Moutawakil [1] together with common (E.A.) property due to $\mathrm{Liu}, \mathrm{Wu}$ and $\mathrm{Li}$ [2].
\end{abstract}

Index Terms- Fuzzy metric space, R-weakly commuting mappings, Property (E.A.), Common (E.A) property, Implicit relation

\section{INTRODUCTION}

In 1986, Jungck [3] introduced the notion of compatible maps for a pair of self mappings. However, the study of common fixed points of non-compatible maps is also very interesting. Aamri and El. Moutawakil [1] generalized the concept of noncompatibility by defining the notion of property (E.A) and in 2005, $\mathrm{Liu}, \mathrm{Wu}$ and $\mathrm{Li}$ [2] defined common (E.A) property in metric spaces and proved common fixed point theorems under strict contractive conditions. Jungck and Rhoades [4] initiated the study of weakly compatible maps in metric space and showed that every pair of compatible maps is weakly compatible but reverse is not true. In the literature, many results have been proved for contraction maps satisfying property
(E.A.) in different settings such as probabilistic metric spaces [5, 6]; fuzzy metric spaces $[7,8,9,10,11]$.

In this paper, employing the common (E.A) property, we prove a common fixed theorem for $R$-weakly commuting mappings via an implicit relation in fuzzy metric space.

\section{PReliminaries}

Definition 2.1. [12] A binary operation * : [0,1] $\times[0,1]$ $\rightarrow[0,1]$ is continuous $t$-norm if $*$ satisfies the following conditions:

(i) * is commutative and associative;

(ii) * is continuous;

(iii) $a * 1=a$ for all $a \in[0,1]$;

(iv) $a * b \leq c * d$ whenever $a \leq c$ and $b \leq d$ for all $a, b, c, d \in[0,1]$.

Kramosil I and Michalek J.[13] introduced the concept of fuzzy metric spaces as follows:

Definition 2.2[13]: The 3-tuple $(X, M, *)$ is called a fuzzy metric space (shortly, FM-space) if $X$ is an arbitrary set, $*$ is a continuous $t$-norm and $M$ is a fuzzy set in $X^{2} \times[0, \infty)$ satisfying the following conditions:

$(\mathrm{FM}-1) M(x, y, 0)=0$,

(FM-2) $M(x, y, t)=1$, for all $t>0$ if and only if $x=y$, $(\mathrm{FM}-3) M(x, y, t)=M(y, x, t)$, 
$(\mathrm{FM}-4) \mathrm{M}(\mathrm{x}, \mathrm{y}, \mathrm{t}) * \mathrm{M}(\mathrm{y}, \mathrm{z}, \mathrm{s}) \leq \mathrm{M}(\mathrm{x}, \mathrm{z}, \mathrm{t}+\mathrm{s})$

(Triangular inequality) and

$(\mathrm{FM}-5) M(x, y,):.[0,1) \rightarrow[0,1]$ is left continuous for all $x, y, z \in X$ and $s, t>0$.

Note that $M(x, y, t)$ can be thought of as the degree of nearness between $\mathrm{x}$ and $\mathrm{y}$ with respect to $t$.

We can fuzzify examples of metric spaces into fuzzy metric spaces in a natural way:

Let $(X, d)$ be a metric space. Define $a * b=a+b$ for all $a, b$ in $X$. Define $M(x, y, t)=t /(t+d(x, y))$ for all $x$, $y$ in $X$ and $t>0$. Then $(X, M, *)$ is a fuzzy metric space and this fuzzy metric induced by a metric $d$ is called the Standard fuzzy metric.

Consider $\mathrm{M}$ to be a fuzzy metric space with the following condition:

(FM-6) $\lim _{t \rightarrow \infty} M(x, y, t)=1$ for all $x, y$ in $X$ and $t>0$.

Definition 2.3[13]: Let $(X, M, *)$ be fuzzy metric space. Then

(a) a sequence $\left\{x_{n}\right\}$ in $X$ is said to be Cauchy sequence if, for all $t>0$ and $p>0$,

$$
\lim _{n \rightarrow \infty} M\left(x_{n+p}, x_{n}, t\right)=1
$$

and

(b) a sequence $\left\{x_{n}\right\}$ in $X$ is said to be convergent to a point $x \in X$ if, for all $t>0$,

$\lim _{n \rightarrow \infty} M\left(x_{n}, x, t\right)=1$.

Definition 2.4[13]: A fuzzy metric space $(X, M, *)$ is said to be complete if and only if every Cauchy sequence in $X$ is convergent.

Example 2.1[13]: Let $X=\{1 / n: n \in N\} \cup\{0\}$ and let $*$ be the continuous t-norm and defined by $a^{*} b=a b$ for all $a, b \in[0,1]$. For each $t>0$ and $x, y \in X$, define $\mathrm{M}$, by

$$
M(x, y, t)=\left\{\begin{array}{cc}
\frac{\mathrm{t}}{\mathrm{t}+|\mathrm{x}-\mathrm{y}|}, & \mathrm{t}>0 \\
0 & \mathrm{t}=0
\end{array}\right.
$$

Clearly, $(X, M, *)$ is complete fuzzy metric space.

Definition 2.5[11]: A pair of self mappings $(A, S)$ of a fuzzy metric space $(X, M, *)$ is said to be commuting if

$M(A S x, S A x, t)=1$ for all $x \in X$.

Definition 2.6[11]: A pair of self mappings $(A, S)$ of a fuzzy metric space $(X, M, *)$ is said to be weakly commuting if $M(A S x, S A x, t) \geq M(A x, S x, t)$ for all $x \in X$ and $t>0$.

Definition 2.7[11]: A pair of self mappings $(A, S)$ of a fuzzy metric space $(X, M, *)$ is said to be compatible if $\lim _{n \rightarrow \infty} M\left(A S x_{n}, S A x_{n}, t\right)=1$ for all $t>0$, whenever $\left\{x_{n}\right\}$ is a sequence in $X$ such that $\lim _{n \rightarrow \infty} A x_{n}=\lim _{n \rightarrow \infty}$ $S x_{n}=u$ for some $u$ in $X$.

Definition 2.8[10]: Let $(X, M, *)$ be a fuzzy metric space. $A$ and $S$ be self maps on $X$. A point $x$ in $X$ is called a coincidence point of $A$ and $S$ iff $A x=S x$. In this case, $w=A x=S x$ is called a point of coincidence of $A$ and $S$.

Definition 2.9[10]: A pair of self mappings $(A, S)$ of a fuzzy metric space $(X, M, *)$ is said to be weakly compatible if they commute at the coincidence points i.e., if $A u=S u$ for some $u \in X$, then $A S u=S A u$.

It is easy to see that two compatible maps are weakly compatible but converse is not true.

Definition 2.10 [1]: A pair of self mappings $(A, S)$ of a fuzzy metric space $(X, M, *)$ is said to satisfy the property (E.A) if there exist a sequence $\left\{x_{n}\right\}$ in $X$ such that $\lim _{n \rightarrow \infty} A x_{n}=\lim _{n \rightarrow \infty} S x_{n}=z$ for some $z \in X$.

Example 2.2[1]: Let $\mathrm{X}=[0, \infty)$. Consider $(X, M, *)$ be a fuzzy metric space as in Example 2.1. 
Define $A, S: X \rightarrow X$ by $A x=\frac{\mathrm{x}}{5}$ and $S x=\frac{2 \mathrm{x}}{5}$ for all $x \in X$. Clearly, for sequence $\left\{x_{n}\right\}=\{1 / n\}, A$ and $S$ satisfies property (E.A).

Definition 2.11 [2]: Two pairs $(A, S)$ and $(B, T)$ of self mappings of a fuzzy metric space $(X, M, *)$ are said to satisfy the common (E.A) property if there exist two sequences $\left\{x_{n}\right\}$ and $\left\{y_{n}\right\}$ in $X$ such that $\lim _{n \rightarrow \infty} A x_{n}=\lim _{n \rightarrow \infty} S x_{n}=\lim _{n \rightarrow \infty} B y_{n}=\lim _{n \rightarrow \infty} T y_{n}=z$

for some $z \in X$.

Example 2.3[2]: Let $X=[-1,1]$. Consider $(X, M, *)$ be a fuzzy metric space as in Example 2.1. Define self mappings $A, \quad B, \quad S$ and $T$ on $X$ as $A x=x / 3, B x=-x / 3, S x=x, T x=-x$ for all $x \in X$. Then, with sequences $\left\{x_{n}\right\}=\{1 / n\}$ and $\left\{y_{n}\right\}=\{-1 / n\}$ in $X$, one can easily verify that $\lim _{n \rightarrow \infty} A x_{n}=\lim _{n \rightarrow \infty} S x_{n}=\lim _{n \rightarrow \infty} B y_{n}=\lim _{n \rightarrow \infty} T y_{n}=0$

Therefore, pairs $(A, S)$ and $(B, T)$ satisfies the common (E.A.) property.

Definition 2.12[11]: A pair of self mappings $(A, S)$ of a fuzzy metric space $(X, M, *)$ is said to be $R$-weakly commuting if there exist $R>0$ such that

$M(A S u, S A u, t) \geq M(A u, S u, t / R)$.

\section{MAIN RESULTS}

Implicit relations play important role in establishing of common fixed point results.

Let $\mathrm{M}_{4}$ be the set of all real continuous functions $\phi:[0,1]^{4} \rightarrow \mathrm{R}$, non-decreasing in the first argument and satisfying the following conditions:

(A) $\phi(u, 1, u, 1) \geq 0 \Rightarrow u \geq 1$,

(B) $\phi(u, 1,1, u) \geq 0 \Rightarrow u \geq 1$
(C) $\phi(u, u, 1,1) \geq 0 \Rightarrow u \geq 1$

Example 3.1: Define $\quad \phi:[0,1]^{4} \rightarrow R \quad$ as $\phi\left(t_{1}, t_{2}, t_{3}, t_{4}\right)=14 t_{1}-12 t_{2}+6 t_{3}-8 t_{4}$. Clearly, $\phi$ satisfies all conditions (A), (B) and (C). Therefore, $\phi \in M_{4}$.

We begin with following observation:

Lemma 3.1: Let $\left\{A_{i}\right\}, \mathrm{S}$ and $\mathrm{T}$ be self mappings of a fuzzy metric space $(X, M, *)$ satisfying the following:

(3.1) the pair $\left(A_{0}, T\right)$ satisfies the E.A. property;

(3.2) for any $\mathrm{x}, \mathrm{y}$ in $\mathrm{X}, \phi$ in $\mathrm{M}_{4}$ and and for all $t>0$ there exists $k \in(0,1)$ such that,

$\phi\left(\begin{array}{l}M\left(A_{i} x, A_{0} y, k t\right), M(S x, T y, t), \\ M\left(S x, A_{i} x, t\right), M\left(T y, A_{0} y, k t\right)\end{array}\right) \geq 0 ;$

(3.3) $A_{i}(X) \subset T(X)$ or $A_{0}(X) \subset S(X)$.

Then the pairs $\left(A_{i}, S\right)$ and $\left(A_{0}, T\right)$ share the common (E.A.) property.

Proof: As the pair $\left(A_{0}, T\right)$ satisfies E.A. property, then there exist a sequence $\left\{x_{n}\right\}$ in $\mathrm{X}$ such that $\lim _{n \rightarrow \infty} A_{0} x_{n}=\lim _{n \rightarrow \infty} T x_{n}=z$ for some $z \in X$. Since $A_{0}(X) \subset S(X)$, hence for each $\left\{x_{n}\right\}$, there exist $\left\{y_{n}\right\}$ in $\mathrm{X}$ such that $A_{0} x_{n}=S y_{n}$. Therefore, $\lim _{n \rightarrow \infty} A_{0} x_{n}=\lim _{n \rightarrow \infty} S y_{n}=\lim _{n \rightarrow \infty} T x_{n}=z$. Now, we claim that $\lim _{n \rightarrow \infty} A_{i} y_{n}=z$.

Suppose that $\lim _{n \rightarrow \infty} A_{i} y_{n} \neq z$, then applying inequality (3.2), we obtain

$\phi\left(\begin{array}{l}M\left(A_{i} y_{n}, A_{0} x_{n}, k t\right), M\left(S y_{n}, T x_{n}, t\right), \\ M\left(S y_{n}, A_{i} y_{n}, t\right), M\left(T x_{n}, A_{0} x_{n}, k t\right)\end{array}\right) \geq 0$ 
which on making $n \rightarrow \infty$ reduces to

$$
\phi\left(\begin{array}{l}
M\left(\lim _{n \rightarrow \infty} A_{i} y_{n}, z, k t\right), M(z, z, t), \\
M\left(\lim _{n \rightarrow \infty} A_{i} y_{n}, z, t\right), M(z, z, k t)
\end{array}\right) \geq 0
$$

As $\phi$ is non-decreasing in the first argument, we have

$\phi\left(\begin{array}{l}M\left(\lim _{n \rightarrow \infty} A_{i} y_{n}, z, t\right), 1, \\ M\left(\lim _{n \rightarrow \infty} A_{i} y_{n}, z, t\right), 1\end{array}\right) \geq 0$

Using (B), we get $M\left(\lim _{n \rightarrow \infty} A_{i} y_{n}, z, t\right) \geq 1$. Hence $M\left(\lim _{n \rightarrow \infty} A_{i} y_{n}, z, t\right)=1$. Therefore, $\lim _{n \rightarrow \infty} A_{i} y_{n}=z$. Hence, the pairs $\left(A_{i}, S\right)$ and $\left(A_{0}, T\right)$ share the common (E.A.) property.

Theorem 3.1: Let $\left\{A_{i}\right\}, S$ and $T$ be self mappings of a fuzzy metric space $(X, M, *)$ satisfying the conditions (3.1), (3.2), (3.3) of lemma 3.1 and the pairs $\left(A_{i}, S\right)$ and $\left(A_{0}, T\right)$ are $R$-weakly commuting. If range of one of $S$ and $T$ is closed subspace of $X$ then $\left\{A_{i}\right\}, S$ and $T$ have a unique common fixed point.

Proof: By lemma 3.1, the pairs $\left(A_{i}, S\right)$ and $\left(A_{0}, T\right)$ share the common (E.A.) property, i.e. there exist two sequences $\left\{x_{n}\right\}$ and $\left\{y_{n}\right\}$ in $X$ such that $\lim _{n \rightarrow \infty} A x_{n}=\lim _{n \rightarrow \infty} S x_{n}=\lim _{n \rightarrow \infty} B y_{n}=\lim _{n \rightarrow \infty} T y_{n}=z \quad$ for some $z \in X$. Suppose that $S(X)$ is a closed subset of $X$, therefore, there exists a point $u \in X$ such that $z=S u$. We claim that $A_{i} u=z$. If $A_{i} u \neq z$, then by (3.2), take $x=u, y=x_{n}$,

$$
\phi\left(\begin{array}{l}
M\left(A_{i} u, A_{0} x_{n}, k t\right), M\left(S u, T x_{n}, t\right), \\
M\left(S u, A_{i} u, t\right), M\left(T x_{n}, A_{0} x_{n}, k t\right)
\end{array}\right) \geq 0
$$

$$
\begin{aligned}
& n \rightarrow \infty \\
& \phi\left(\begin{array}{l}
M\left(A_{i} u, z, k t\right), M(z, z, t), \\
M\left(z, A_{i} u, t\right), M(z, z, k t)
\end{array}\right) \geq 0 \\
& \phi\left(M\left(A_{i} u, z, k t\right), 1, M\left(A_{i} u, z, t\right), 1\right) \geq 0
\end{aligned}
$$

As $\phi$ is non-decreasing in the first argument, we have

$\phi\left(M\left(A_{i} u, z, t\right), 1, M\left(A_{i} u, z, t\right), 1\right) \geq 0$

and using (A), we get $M\left(A_{i} u, z, t\right) \geq 1$.

Hence $M\left(A_{i} u, z, t\right)=1$. Therefore, $A_{i} u=z=S u$ which shows that $\mathrm{u}$ is a coincidence point of the pair $(A$, S).

Since, $A_{i}(X) \subset T(X)$, there exists $v \in X$ such that $T v=z=A_{i} u=S u$. Now, we show that $A_{0} v=z$.

If $\mathrm{A}_{0} \mathrm{~V} \neq \mathrm{z}$, then by using inequality (3.2), take $x=$ $y_{n}, y=v$, we have

$\phi\left(\begin{array}{l}M\left(A_{i} y_{n}, A_{0} v, k t\right), M\left(S y_{n}, T v, t\right), \\ M\left(S y_{n}, A_{i} y_{n}, t\right), M\left(T v, A_{0} v, k t\right)\end{array}\right) \geq 0$

$n \rightarrow \infty$

$\phi\left(\begin{array}{l}M\left(z, A_{0} v, k t\right), M(z, z, t), M(z, z, t), \\ M\left(z, A_{0} v, k t\right)\end{array}\right) \geq 0$

Using (B), we get $M\left(z, A_{0} v, k t\right) \geq 1$.

Hence $M\left(z, A_{0} v, k t\right)=1$. Therefore, $A_{0} v=z=T v$ which shows that $v$ is a coincidence point of the pair $\left(A_{0}, T\right)$. Since, $A_{i}$ and $S$ are pointwise $R$-weakly commuting, there exist $R>0$ such that

$M\left(A_{i} S u, S A_{i} u, t\right) \geq M\left(A_{i} u, S u, t / R\right)=1$.

This gives, $A_{i} S u=S A_{i} u=A A_{i} u=S S u$.

Similarly, as $A_{0}$ and $T$ are pointwise $R$-weakly commuting, we have $A_{0} T v=T A_{0} v$ and $A_{0} T v=T A_{0} v=$ $A_{0} A_{0} v=T T v$.

Take $x=A_{i} u, y=v$ in (3.2), we have 


$$
\begin{aligned}
& \phi\left(\begin{array}{l}
M\left(A_{i} A_{i} u, A_{0} v, k t\right), M\left(S A_{i} u, T v, t\right), \\
M\left(S A_{i} u, A_{i} A_{i} u, t\right), M\left(T v, A_{0} v, k t\right)
\end{array}\right) \geq 0 \\
& \phi\left(M\left(A_{i} A_{i} u, A_{i} u, k t\right), M\left(S S u, A_{i} u, t\right), 1,1\right) \geq 0 ;
\end{aligned}
$$

As $\phi$ is non-decreasing in the first argument, we have

$\phi\left(M\left(A_{i} A_{i} u, A_{i} u, t\right), M\left(S S u, A_{i} u, t\right), 1,1\right) \geq 0$

Using (C), we get $M\left(A_{i} A_{i} u, A_{i} u, t\right) \geq 1$.

This gives, $M\left(A_{i} A_{i} u, A_{i} u, t\right)=1$.

Therefore, $A_{i} A_{i} u=A_{i} u$. Hence, $A_{i} z=z=S z$.

Similarly, by putting, $y=A_{0} v, x=u$ in (3.2), we get $\phi\left(\begin{array}{l}M\left(A_{i} u, A_{0} A_{0} v, k t\right), M\left(S u, T A_{0} v, t\right), \\ M\left(S u, A_{i} u, t\right), M\left(T A_{0} v, A_{0} A_{0} v, k t\right)\end{array}\right) \geq 0$ $\phi\left(M\left(A_{0} v, A_{0} A_{0} v, k t\right), M\left(A_{0} v, A_{0} A_{0} v, t\right), 1,1\right) \geq 0 ;$

As $\phi$ is non-decreasing in the first argument, we hav

$$
\begin{aligned}
& \phi\left(M\left(A_{0} v, A_{0} A_{0} v, t\right), M\left(A_{0} v, A_{0} A_{0} v, t\right), 1,1\right) \geq 0 ; \\
& M\left(A_{0} v, A_{0} A_{0} v, t\right) \geq 1 ;
\end{aligned}
$$

therefore, $M\left(A_{0} v, A_{0} A_{0} v, t\right)=1$;

This gives, $A_{0} v=A_{0} A_{0} v$.

$$
A_{0} z=z=T z
$$

Hence, $A_{i} z=A_{0} z=S z=T z$, and $z$ is common fixed point of $A_{i}, A_{0}, S$ and $T$.

Uniqueness: Let $z$ and $w$ be two common fixed points of $A_{i}, A_{0}, S$ and $T$. If $z \neq w$, then by using inequality (3.2), we have

$$
\begin{aligned}
& \phi\left(\begin{array}{l}
M\left(A_{i} z, A_{0} w, k t\right), M(S z, T w, t), \\
M\left(S z, A_{i} z, t\right), M\left(T w, A_{0} w, k t\right)
\end{array}\right) \geq 0, \\
& \phi\left(\begin{array}{l}
M(z, w, k t), M(z, w, t), \\
M(z, z, t), M(w, w, k t)
\end{array}\right) \geq 0, \\
& \phi(M(z, w, k t), M(z, w, t), 1,1) \geq 0, \\
& \phi(M(z, w, t), M(z, w, t), 1,1) \geq 0 .
\end{aligned}
$$

Using (C) and (F), we have $M(z, w, t) \geq 1$.

Hence, $M(z, w, t)=1$.

Therefore, $z=w$

By choosing $A_{i}, A_{0}, S$ and $T$ suitably, one can derive corollaries involving two or more mappings. As a sample, we deduce the following natural result for a pair of self mappings by setting $A_{i}=A_{0}$ and $T=S$ in above theorem:

Corollary 3.1. Let $\left\{A_{i}\right\}$ and $S$ be self mappings of a fuzzy metric space $(X, M, *)$ satisfying the following:

(3.4) the pair $\left(A_{i}, S\right)$ satisfies the E.A. property;

(3.5) for any $x, y$ in $X, \phi$ in $M_{4}$ and for all $t>0$,

$\phi\left(\begin{array}{l}M\left(A_{i} x, A_{i} y, k t\right), M(S x, S y, t), \\ M\left(S x, A_{i} x, t\right), M\left(S y, A_{i} y, t\right)\end{array}\right) \geq 0$

(3.6) $S(X)$ is a closed subset of $X$.

Then, $\left\{A_{i}\right\}$ and $S$ have a point of coincidence each. Moreover, if the pairs $\left(A_{i}, S\right)$ is weakly compatible, then $\left\{A_{i}\right\}$ and $S$ have a unique common fixed point.

By taking $A_{i}=A_{0}=A$ and $T=S$ in theorem 3.1, we get

Corollary 3.2. Let $A$ and $S$ be self mappings of a fuzzy metric space $(X, M, *)$ satisfying the following:

(3.7) the pair $(A, S)$ satisfies the E.A. property;

(3.8) for any $x, y$ in $X, \phi$ in $\mathrm{M}_{4}$ and for all $t>0$,

$\phi\left(\begin{array}{l}M(A x, A y, k t), M(S x, S y, t), \\ M(S x, A x, t), M(S y, A y, t)\end{array}\right) \geq 0 ;$

(3.8) $S(X)$ is a closed subset of $X$.

Then, $A$ and $S$ have a point of coincidence each. Moreover, if the pairs $(A, S)$ is weakly compatible, then $A$ and $S$ have a unique common fixed point.

The following example illustrates Theorem 3.1. 
Example 3.2. Let $(X, M, *)$ be a fuzzy metric space where $X=[0,2)$ and define $\phi:[0,1]^{4} \rightarrow \mathrm{R}$ as $\phi\left(t_{1}, t_{2}, t_{3}, t_{4}\right)=14 t_{1}-12 t_{2}+6 t_{3}-8 t_{4}$. Clearly, $\phi$ satisfies all conditions (A), (B) and (C). Therefore, $\phi \in M_{4}$. Define $A, B, S$ and $T$ by

$A_{i} x=A_{0} x=1$,

$S(x)=\left\{\begin{array}{ll}1 & x \in Q \\ \frac{2}{3} & x \notin Q,\end{array}, \quad T(x)= \begin{cases}1 & x \in Q \\ \frac{1}{3} & x \notin Q\end{cases}\right.$ and $M(x, y, t)=\frac{t}{t+|x-y|}$ for all $x, y$ in $X=[0$, 2 ) and $t>0$.Then with sequences $\left\{x_{n}=\frac{1}{n}\right\}$ and $\left\{y_{n}=\frac{-1}{n}\right\}$ in $\mathrm{X}$, we have

$\lim _{n \rightarrow \infty} A_{i} x_{n}=\lim _{n \rightarrow \infty} S x_{n}=\lim _{n \rightarrow \infty} A_{0} y_{n}=\lim _{n \rightarrow \infty} T y_{n}=1$ in $X$

which shows that pairs $\left(A_{i}, S\right)$ and $\left(A_{0}, T\right)$ share the common (E.A.) property. By a routine calculation, one can verify the condition (3.2). Thus, all the conditions of Theorem 3.1 are satisfied and $x=1$ is the unique common fixed point of $A_{i}, A_{0}, S$ and $T$.

\section{ACKNOWLEDGMENT}

The author wish to thank Dr. Sunny Chauhan, Dr Suneel Kumar and the referees for their very helpful suggestions and many kind comments.

\section{REFERENCES}

[1] M. Aamri and D. El Moutawakil, Some new common fixed point theorems under strict contractive conditions, J. Math. Anal. Appl., 270 (2002), 181-188.
[2] W. Lui, J. Wu and Z. Li, Common fixed points of single-valued and multivalued maps, Int. J. Math. Math. Sci, 19 (2005), 3045-3055.

[3] G. Jungck, Compatible mappings and common fixed points, Internat. J. Math. Math. Sci., 9 (1986), 771-779.

[4] G. Jungck and B.E. Rhoades, Fixed points for set valued functions without continuity, Indian J. Pure Appl. Math., 29 (1998), 227-238.

[5] J. X. Fang and Y. Gao, Common fixed point theorems under strict contractive conditions in Menger spaces, Nonlinear Analysis, 70 (1) (2009), 184-193.

[6] M. Imdad, M. Tanveer and M. Hasan, Some common fixed point theorems in Menger PMspaces, Fixed Point Theory and Applications, Volume 2010, Article ID 819269, 14 pages.

[7] S. Kumar and B. Fisher, A common fixed point theorem in fuzzy metric space using property (E. A.) and implicit relation, Thai J. Math., 8 (3) (2010), 439-446.

[8] D. Mihet, Fixed point theorems in fuzzy metric spaces using property E.A., Nonlinear Analysis, 73 (7) (2010), 2184-2188.

[9] S.S. Bhatia, S. Manro and S. Kumar, Fixed point theorem for weakly compatible maps using E.A. Property in Fuzzy metric spaces satisfying contractive condition of Integral type, Int. J. Contemp. Math. Sciences, 5 (51)(2010), $2523-2528$.

[10] S. Manro, A Common Fixed Point Theorem for Weakly Compatible Maps Satisfying Property (E.A.) in Fuzzy Metric Spaces using Strict Contractive Condition. ARPN Journal of Science and Technology, 2(4) (2012), 367370. 
[11] S. Manro, S.S. Bhatia and S. Kumar, Common fixed point theorems in fuzzy metric spaces, Annals of Fuzzy Mathematics and Informatics, 3(1)(2012), 151- 158.

[12] K. Menger, Statistical metrics, Proc. Nat. Acad. Sci. (USA), 28 (1942), 535-537.

[13] I. Kramosil and J. Michalek, Fuzzy metric and statistical spaces, Kybernetica, 11(1975), 336344.

Saurabh Manro is the Research Scholar in School of Mathematics and Computer Applications, Thapar University, Patiala (Punjab), India. He completed M.Sc (Mathematics) in year 2004 from Punjab University, Chandigarh. He is pursuing Ph.D under the kind supervision of Dr. S.S. Bhatia and Dr. Sanjay Kumar. His areas of research include Fixed point theorem in various abstract spaces like Menger spaces, Probabilistic Metric spaces, Fuzzy Metric spaces, Intuitionistic Fuzzy Metric spaces, G- Metric spaces and its applications. He has published many research papers in national / international papers till now. Some papers are ready to be published. 